\title{
Bargaining under monotonicity constraints
}

\author{
P. Jean-Jacques Herings ${ }^{1}$ • A. Predtetchinski ${ }^{1}$
}

Received: 29 August 2014 / Accepted: 14 May 2015 / Published online: 31 May 2015 (C) The Author(s) 2015. This article is published with open access at Springerlink.com

\begin{abstract}
We study unanimity bargaining on the division of a surplus in the presence of monotonicity constraints. The monotonicity constraints specify a complete order on the players, which has to be respected by the shares in the surplus the players obtain in any bargaining outcome. A player higher in the order should not receive a lower share of the surplus. We analyze the resulting subgame perfect equilibria in stationary strategies and show that they are characterized by the simpler notion of bargaining equilibrium. Bargaining equilibria are shown to be unique and to have the property that players ranked strictly higher obtain strictly higher shares in the surplus. The key question is whether the bargaining advantage of a higher-ranked player persists when the probability of breakdown of bargaining tends to zero. We argue that such is not the case by showing that bargaining equilibria have a unique limit equal to an equal division of the surplus. It then follows that the limit also coincides with the Nash bargaining solution for this problem.
\end{abstract}

Keywords Non-cooperative bargaining · Monotonicity constraints · Subgame perfect equilibrium $\cdot$ Nash bargaining solution

JEL Classification C72 $\cdot$ C78 $\cdot$ D63

The authors would like to thank the Netherlands Organization for Scientific Research (NWO) for financial support.

$凶 \quad$ P. Jean-Jacques Herings

P.Herings@maastrichtuniversity.nl

A. Predtetchinski

A.Predtetchinski@maastrichtuniversity.nl

1 Department of Economics, Maastricht University, P.O. Box 616, 6200 MD Maastricht,

The Netherlands 


\section{Introduction}

The path-breaking paper by Rubinstein (1982) on alternating offers bargaining has spurred an extensive literature to explain the crucial factors that determine the division of a surplus among a group of players. It is common in the literature to assume that any division of the surplus among the players can be proposed. However, in many applications there are monotonicity constraints on the proposed shares that have to be respected, an important aspect which has been disregarded in the bargaining literature so far.

Very often these monotonicity constraints are dictated by custom. If a team carries out a joint project, it would be customary that the team leader should not be paid less than the other team members. Similarly, if a team consists of workers with differing experience, then more experienced workers would not be paid less than less experienced workers. Although in the first example, it would technically be possible to pay the team leader less than the other team members, making such a proposal could be so inappropriate that it would severely affect the rest of the bargaining process and therefore essentially such proposals are ruled out. In the second example, legal constraints or collective labor agreements could literally prevent proposals from violating particular monotonicity constraints.

Monotonicity constraints also result often from incentive compatibility considerations. When workers in a team are endowed with unobservable efficiency units of labor, but produce observable output, then incentive compatibility would require that workers with higher output should not receive a lower share of the total surplus generated. Similar monotonicity constraints arise in the theory of income taxation following Mirrlees (1971), which we used as the main motivation in a previous version of this paper, see Herings and Predtetchinski (2011b).

Monotonicity constraints also often serve as desirable normative principles, for instance in bankruptcy problems as studied in the seminal paper by O'Neill (1982). The bankruptcy problem is based on an example in the Talmud, where the estate left behind by a deceased man is worth less than the claims of his wives. The question is how the estate should be divided among the claimants. Based on Aumann and Maschler (1985), the solution to a bankruptcy problem is said to be order-preserving if a person with a claim at least as large as another person receives at least as much as the other person. Order preservation corresponds exactly to the monotonicity constraints we study in this paper and is generally met by solutions proposed for bankruptcy problems like the proportional rule, the constrained equal award rule, or the Talmud rule, see Thomson (2003) for an overview of the literature.

We study unanimity bargaining on the division of a surplus in the presence of monotonicity constraints. Monotonicity constraints specify a complete order on the players, which has to be respected by the shares in the surplus offered to them in any proposal. In the presence of monotonicity constraints, an equal division of the surplus is still feasible and yields the lowest possible payoffs to the players in the highest indifference class. The most extreme proposal in the other direction is to propose zero to all players except those in the highest indifference class. Although such a proposal is feasible, it will not be proposed by any player, not even the ones ranked highest, since it is surely rejected by the other players. A proposal involving an equal division of 
the surplus will also not be proposed by the highest ranked players. The monotonicity constraints imply that such players receive at least the average surplus in all proposals and strictly more if they are proposing themselves.

We analyze the subgame perfect equilibria in stationary strategies of the model and are mainly interested in the question to what extent players higher in the order can exploit their favorable bargaining position, in particular for the case where the bargaining breakdown probability tends to zero.

Subgame perfect equilibria in stationary strategies can be characterized by the more elementary notion of a bargaining equilibrium. A bargaining equilibrium is a tuple of proposals by the various players which are all unanimously accepted. Moreover, the proposal of a player is the one giving him the highest payoff among all proposals that are accepted by all players and that obey the monotonicity constraints.

Consider a bargaining equilibrium proposal by a particular player. All lower-ranked players are offered their reservation payoff derived from the bargaining equilibrium. To avoid rejection by higher-ranked players, also those have to be promised at least their reservation payoff. Monotonicity constraints may force the proposing player to make a proposal strictly above their reservation payoff. In general, the bargaining equilibrium proposal is such that all players ranked higher than the proposer up to some rank are proposed the same amount as the proposer keeps for himself, and players ranked even higher are proposed their reservation payoffs.

Monotonicity constraints would be satisfied by the set of alternatives as considered in the more general frameworks of Banks and Duggan (2000) and Duggan (2011), although the specification of the voting stages differs since they consider simultaneous voting and restrict attention to equilibria with stage-undominated voting strategies. By Theorem 2 in Banks and Duggan (2000), there exists such an equilibrium that is in pure stationary strategies. In contrast to the results on the existence of stationary equilibria, conditions for the uniqueness of stationary equilibria found in the literature do not apply to our setup.

General conditions for the uniqueness of subgame perfect equilibria in stationary equilibria in unanimity bargaining games are given by Merlo and Wilson (1995). However, the monotonicity constraints we impose imply that their assumptions on the set of feasible alternatives are violated. Other uniqueness results that have been derived in the context of one-dimensional bargaining problems, see Imai and Salonen (2000), Cho and Duggan (2003), Cardona and Ponsatí (2007), and Herings and Predtetchinski (2010) or in the context of legislative bargaining problems as in Eraslan and McLennan (2013) do not apply either. In the presence of monotonicity constraints, we can show that bargaining equilibria are unique by a contraction argument. As a by-product of this result, we obtain equilibrium existence.

Consider a sequence of breakdown probabilities converging to zero and consider the corresponding sequence of bargaining equilibria. It follows from a standard argument that all players make the same proposal in the limit. We are interested in the uniqueness and the characterization of the limit proposal. The literature on multilateral bargaining with unanimous agreement has shown convergence of bargaining equilibrium proposals to the Nash bargaining solution, see Hart and Mas-Colell (1996), Laruelle and Valenciano (2007), Miyakawa (2008), Kultti and Vartiainen (2010), and Britz et al. (2010). Unfortunately, all these papers need differentiability assumptions with respect 
to the set of feasible alternatives, an assumption that is clearly violated in the presence of monotonicity constraints. Moreover, Kultti and Vartiainen (2010) and Herings and Predtetchinski (2011a) present examples where in the absence of differentiability assumptions, sequences of bargaining equilibria may have a limit different from the Nash bargaining solution and may have multiple limits.

For the cases with two or three players and a strict order on the players, we calculate bargaining equilibria explicitly and verify the uniqueness of the limit proposal. For these cases, it corresponds to the equal division of the surplus, although for the threeplayer case this outcome is not proposed by any player for sufficiently low values of the bargaining breakdown probability, but is only reached in the limit. The equal division of the surplus is also the outcome predicted by an application of the Nash bargaining solution to our problem. We demonstrate that also in the case with an arbitrary number of players and an arbitrary monotonicity constraints, the limit proposal is unique and leads to an equal division of the surplus.

This paper is organized as follows. Section 2 introduces the bargaining procedure and Sect. 3 the notion of bargaining equilibrium. Section 4 characterizes optimal proposals of players, and Sect. 5 demonstrates the uniqueness of bargaining equilibria. Section 6 computes the bargaining equilibrium explicitly for two simple examples and shows that it converges to an equal division of the surplus in the limit when the bargaining breakdown probability converges to zero. Limit equilibria are formally introduced in Sect. 7. Section 8 proves that limit equilibria are unique, lead to an equal division of the surplus, and correspond therefore to the Nash bargaining solution for this problem. Section 9 concludes.

\section{The bargaining procedure}

We study a bargaining game $\Gamma(N, \preceq)$, where a group of players $N=\{1, \ldots, n\}$ bargains about the division of a surplus of size one and $\preceq$ is a complete order on the players. A division of the surplus is a vector of shares $x=\left(x_{1}, \ldots, x_{n}\right)$, where $x_{i}$ is the share in the surplus of player $i$. For a group of players $G \subset N$ and a vector $y \in \mathbb{R}^{n}$, we use the notation $y(G)=\sum_{i \in G} y_{i}$. A group of players $G$ of the form $\{i, i+1, \ldots, j-1, j\}$ for some $i, j \in N$, is denoted $[i, j]$.

The order $\preceq$ specifies the restriction that a higher-ranked player should at least receive the same share in the surplus as a lower-ranked player, so for $i, j \in N$, if $i \preceq j$, then $x_{i} \leq x_{j}$. Without loss of generality, we assume that $1 \preceq 2 \preceq \cdots \preceq n$. Notice that two or more players may belong to the same indifference class. The indifference class of player $i$ is denoted by the possibly degenerate interval $\left[\ell_{i}, u_{i}\right]$, so the collection $\left\{\left[\ell_{i}, u_{i}\right] \mid i \in N\right\}$ is a partition of $[1, n]$. We denote the set of all lower bounds by $L=\left\{\ell_{i} \mid i \in N\right\}$ and upper bounds by $U=\left\{u_{i} \mid i \in N\right\}$.

The set $X$ of feasible divisions of the surplus is then equal to

$$
X=\left\{x \in \mathbb{R}_{+}^{n} \mid x(N)=1, \forall i, j \in N \text { such that } i \preceq j, x_{i} \leq x_{j}\right\} .
$$

Two extreme efficient divisions of the surplus are given by equal sharing where $x_{i}=$ $1 / n$ for all players $i$ on the one hand and the least egalitarian division where $x_{i}=0$ 
for every $i \in N$ such that $i \prec n$, and the surplus is shared equally among all players in the indifference class of player $n$. In case all players different from $n$ are ranked strictly below $n$, we have $x_{i}=0$ for $i \in[1, n-1]$ and $x_{n}=1$.

The bargaining procedure is defined as follows. In each bargaining round $r$, each player has an equal probability to be selected as the proposer. The selected player, say player $i$, makes a proposal $p^{i} \in X$. After observing $p^{i}$, players sequentially decide whether to accept or to reject the proposal in a fixed a priorily chosen order. If all players accept, then the division of the surplus is given by $p^{i}$, leading to a share $p_{j}^{i}$ for player $j \in N$. As soon as some player rejects, bargaining breaks down with probability $1-\delta>0$ and continues with probability $\delta \geq 0$ in bargaining round $r+1$ with the selection of a randomly selected player as the proposer. If no agreement is ever reached, all players receive a share equal to zero.

We analyze the subgame perfect equilibria in stationary strategies (SSPE) of the resulting game. A stationary strategy of player $i, \sigma^{i}=\left(p^{i}, A^{i}\right)$, consists of a proposal $p^{i} \in X$ and an acceptance set $A^{i} \subset X$. The acceptance set consists of those proposals that are accepted by a player. This specification results in a stationary strategy because $p^{i}$ and $A^{i}$ are time and history independent. We write $p=\left(p^{1}, \ldots, p^{n}\right)$ and $A=$ $\left(A^{1}, \ldots, A^{n}\right)$. The social acceptance set consists of the proposals that are accepted by all players and is given by $\cap_{i \in N} A^{i}$. A strategy profile $(p, A)$ is a subgame perfect equilibrium if it induces a Nash equilibrium in every subgame.

A strategy profile $\sigma=\left(\sigma^{i}\right)_{i \in N}$ determines the expected payoff $V^{i}(\sigma)$ for each player $i$ as evaluated at the beginning of the game. This payoff is equal to the expected value of player $i$ 's share in the surplus. Since strategies are stationary, $V^{i}(\sigma)$ is also the continuation payoff of player $i$, the expected payoff as evaluated at the beginning of any bargaining round $r$, and therefore equal to the expected share in the surplus at the beginning of any bargaining round $r$.

A strategy profile $(p, A)$ is called a no-delay strategy profile if $p^{i} \in \cap_{j \in N} A^{j}$ for all $i \in N$.

\section{Bargaining equilibrium}

The concept of SSPE imposes relatively few restrictions on individual acceptance sets. For instance, it may well happen at equilibrium that a player accepts a proposal which is very unfavorable to him in the knowledge that it will be rejected by a player that responds next. To avoid such inessential multiplicity, we introduce a more basic notion of equilibrium, called bargaining equilibrium, which is shown to be essentially equivalent to the notion of SSPE.

The role of these results is auxiliary: They simplify the exposition of the main results on the uniqueness and convergence of equilibria developed in the rest of the paper. For instance, as shown in Sect. 5, bargaining equilibria are unique. Clearly, due to the inessential indeterminacy of the players' acceptance set alluded to above, SSPE are not unique, even though each player makes the same equilibrium proposal in each SSPE. The arguments involved in deriving the equivalence of bargaining equilibria and SSPE are rather standard. We relegate the proofs to the "Appendix". 
In a bargaining equilibrium, a player votes in favor of any proposal that gives the player at least his reservation utility. Consequently, the social acceptance set in a bargaining equilibrium consists of all proposals $x \in X$ that are coordinatewise greater than or equal to the vector of reservation utilities $\delta \sum_{j \in N}(1 / n) p^{j}$.

Definition 3.1 The profile $p \in X^{N}$ is a bargaining equilibrium if, for all $i \in N$, $p^{i} \in \arg \max _{x \in S} x_{i}$, where $S=\left\{x \in X \mid x \geq \delta \sum_{j \in N}(1 / n) p^{j}\right\}$.

Given a bargaining equilibrium $p$, we define the strategy profile $\sigma=(p, A(p))$, where

$$
A^{i}(p)=\left\{x \in X \mid x_{i} \geq \delta \sum_{j \in N}(1 / n) p_{i}^{j}\right\}, \quad i \in N
$$

Notice that at a bargaining equilibrium, every player $i$ makes a proposal $p^{i}$ in the social acceptance set

$$
\cap_{j \in N} A^{j}(p)=\left\{x \in X \mid x \geq \delta \sum_{j \in N}(1 / n) p^{j}\right\}
$$

which coincides with the set $S$ as defined in Definition 3.1. The strategy profile $(p, A(p))$ therefore satisfies the no-delay property. Observe that $\sum_{j \in N}(1 / n) p^{j}$ is equal to the expected division of the surplus in a bargaining equilibrium. According to the individual acceptance set $A^{i}(p)$, a player accepts any proposal giving him a payoff greater than or equal to his expected share of the surplus multiplied by $\delta$. Conditional on being the proposer, every player makes a proposal that maximizes his share among all the proposals in the social acceptance set. Since all proposals $p^{j}$ belong to $X$, and $X$ is convex, it also holds that $\sum_{j \in N}(1 / n) p^{j}$ belongs to $X$. The expected equilibrium share is nonnegative, and players who are higher in the order obtain a higher expected share in the surplus.

Before turning to the existence and uniqueness of bargaining equilibria in Sect. 5, we argue first that bargaining equilibrium is an appropriate concept.

Theorem 3.2 If $p \in X^{N}$ is a bargaining equilibrium, then $(p, A(p))$ is an SSPE.

Theorem 3.3 If $(p, A)$ is an SSPE, then $(p, A)$ is a no-delay strategy profile, and $p$ is a bargaining equilibrium.

It follows from Theorems 3.2 and 3.3 that there is no loss of generality to restrict attention to bargaining equilibria. By Theorem 3.2, every bargaining equilibrium is associated with some SSPE, and the no-delay property presented in Theorem 3.3 implies that for every SSPE there is a payoff equivalent bargaining equilibrium.

\section{Optimal proposals}

In this section, we characterize the solution to player $i$ 's optimization problem of choosing the best proposal within the set of socially acceptable proposals. Before we proceed with the derivations, we recall the logic of the canonical bargaining problem 
where the players have to agree on the division of a dollar. The main feature of dividethe-dollar bargaining is that, in equilibrium, every proposer offers the other players their respective continuation payoffs so that all responders are indifferent between rejecting and accepting an equilibrium proposal.

In the presence of monotonicity constraints, proposals that extract all of the surplus from the responders may not be feasible. In a bargaining equilibrium, the proposal of player $i$ might be strictly preferred by some of the responders to their continuation payoffs. Players ranked strictly below $i$ as well as the players ranked sufficiently far above $i$ are offered exactly their reservation payoffs. Players in the interval $\left[\ell_{i}, m_{i}\right]$, where $m_{i} \geq u_{i}$, are all offered the same share, which weakly exceeds their respective continuation payoffs. The characterization of the set $\left[\ell_{i}, m_{i}\right]$ of players is the main import of this section.

Given an expected equilibrium division of the surplus $y \in X$, player $i$ 's optimization problem is

$$
\begin{aligned}
\operatorname{maximize} & x_{i} \\
\text { subject to } & x \in X, \\
x & \geq \delta y .
\end{aligned}
$$

A solution to this problem is denoted $a^{i}(y) \in X$ and yields the proposed division by player $i$ given an expected equilibrium division $y$. In this section, we argue this solution to be unique.

The set $\{x \in X \mid x \geq \delta y\}$ is compact. The point $\bar{x}=\left(\delta y_{1}+(1-\delta) / n, \ldots, \delta y_{n}+(1-\right.$ $\delta) / n$ ) belongs to $X$, so $X$ is non-empty. Indeed, it holds that $\bar{x}(N)=1$, and for every $i, j \in N$ such that $i \preceq j$ it holds that $\bar{x}_{i}=\delta y_{i}+(1-\delta) / n \leq \delta y_{j}+(1-\delta) / n=\bar{x}_{j}$.

The problem of player $i$ therefore involves the maximization of a continuous function on a non-empty, compact set and therefore has at least one solution. The following proposition is straightforward to verify and is stated without proof.

Proposition 4.1 Consider some $y \in X$. Let $a^{i}(y)$ be a solution to the optimization problem of player $i \in N$. Then there exists a threshold $m_{i} \in\left[u_{i}, n\right] \cap U$ such that

$$
a_{j}^{i}(y)= \begin{cases}\frac{\delta y\left(\left[\ell_{i}, m_{i}\right]\right)+1-\delta}{m_{i}-\ell_{i}+1}, & j \in\left[\ell_{i}, m_{i}\right], \\ \delta y_{j}, & j \in N \backslash\left[\ell_{i}, m_{i}\right],\end{cases}
$$

and

$$
\begin{array}{ll}
\delta y_{m_{i}} \leq a_{m_{i}}^{i}(y)<\delta y_{m_{i}+1}, & \text { if } m_{i}<n, \\
\delta y_{m_{i}} \leq a_{m_{i}}^{i}(y), & \text { if } m_{i}=n .
\end{array}
$$

In an optimal proposal by player $i \in N$, all players in $\left[\ell_{i}, m_{i}\right]$ receive the same share in the surplus, equal to $a_{i}^{i}(y)$. Figure 1 provides a sketch of the share $a_{j}^{i}(y)$ player $i$ offers to player $j$. It is instructive to compare optimal proposals with and without monotonicity constraints. In the absence of monotonicity constraints, player $i$ optimally offers all other players $j$ their respective reservation payoffs, $\delta y_{j}$, and leaves himself a share of $\delta y_{i}+1-\delta$. Under monotonicity constraints, such a proposal 
Fig. 1 Optimal proposals

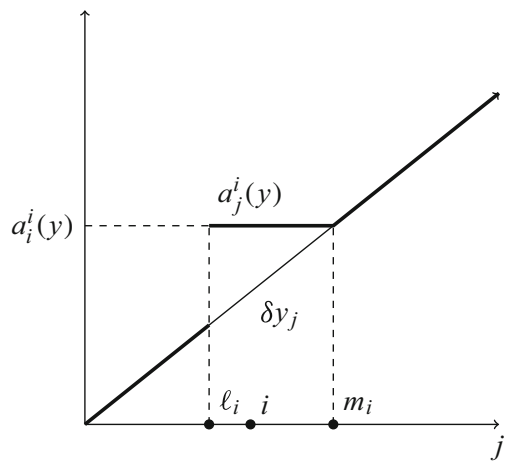

may no longer be feasible, for instance when there is a player $j$ different from $i$ in the same indifference class as player $i$, or when there is a player $j$ with $j \succ i$ and $\delta y_{j}<\delta y_{i}+1-\delta$. Player $i$ is then forced to offer some other players the same share as he receives himself.

Let some $y \in X$ be given. For player $i \in N$, we define the function $g_{i}:\left[u_{i}, n\right] \rightarrow \mathbb{R}$ by

$$
g_{i}(m)=\frac{\delta y\left(\left[\ell_{i}, m\right]\right)+1-\delta}{m-\ell_{i}+1} .
$$

Then $g_{i}(m)$ is equal to the share of player $i$ in the surplus when the players $j \in$ $N \backslash\left[\ell_{i}, m\right]$ are given the share $\delta y_{j}$ and the remainder of the surplus is shared equally between the players in $\left[\ell_{i}, m\right]$. We define the set

$$
M_{i}=\left\{m \in\left[u_{i}, n-1\right] \mid \delta y_{m} \leq g_{i}(m)<\delta y_{m+1}\right\} \cup\left\{m \in\{n\} \mid \delta y_{m} \leq g_{i}(m)\right\} .
$$

It holds that $M_{i} \subset U$. Indeed, if $m \in M_{i}$ is such that $m<n$, then it follows that $m \in U$ from the requirement that $\delta y_{m}<\delta y_{m+1}$. If $m=n$, then it obviously holds that $m \in U$.

From Proposition 4.1, we know that $a_{i}^{i}(y)=g_{i}(m)$ for some $m \in M_{i}$. As Proposition 4.2 below shows, the set $M_{i}$ is a singleton. Therefore, the threshold $m_{i}$ can be characterized as the unique integer $m \in\left[u_{i}, n\right]$ such that ( $m<n$ and $\left.\delta y_{m} \leq g_{i}(m)<\delta y_{m+1}\right)$ or $\left(m=n\right.$ and $\left.\delta y_{m} \leq g_{i}(m)\right)$.

Proposition 4.2 Consider some $y \in X$. For every $i \in N$, the set $M_{i}$ is a singleton.

Proof The proposition is clearly true when $u_{i}=n$. So consider the case where $u_{i}<n$. Some elementary algebra shows that for $m \in\left[u_{i}+1, n\right]$,

$$
g_{i}(m)-g_{i}(m-1)=\frac{\delta y_{m}-g_{i}(m)}{m-\ell_{i}},
$$

so we have that

$$
g_{i}(m) \geq g_{i}(m-1) \text { if and only if } \delta y_{m} \geq g_{i}(m) .
$$


We show next that $M_{i}$ is an interval. Assume that $j$ and $j^{\prime}$ are elements of $M_{i}$ with $j<j^{\prime}$. We prove that the set $M_{i}$ contains the interval $\left[j, j^{\prime}\right]$. It holds that

$$
\delta y_{j^{\prime}-1} \leq \delta y_{j^{\prime}} \leq g_{i}\left(j^{\prime}\right) \leq g_{i}\left(j^{\prime}-1\right)
$$

where the first inequality follows from $y \in X$, the second inequality follows since $j^{\prime} \in$ $M_{i}$, and the third inequality follows from the second inequality and the equivalence in (1). Iterating this argument, we obtain the chain of inequalities

$$
\delta y_{j} \leq \cdots \leq \delta y_{j^{\prime}} \leq g_{i}\left(j^{\prime}\right) \leq \cdots \leq g_{i}(j)
$$

At the same time, we obtain the chain of inequalities

$$
g_{i}\left(j^{\prime}\right) \leq \cdots \leq g_{i}(j)<\delta y_{j+1} \leq \cdots \leq \delta y_{j^{\prime}+1},
$$

where the first tuple of weak inequalities follows from (2), the strict inequality since $j \in M_{i}$, and the last tuple of weak inequalities from $y \in X$. The inequalities in (2) and (3) show that $\left[j, j^{\prime}\right]$ is contained in $M_{i}$.

Finally, we show that the interval $M_{i}$ is degenerate. Suppose that both $j$ and $j+1$ belong to the set $M_{i}$. Then $\delta y_{j} \leq g_{i}(j)<\delta y_{j+1} \leq g_{i}(j+1)$, thus in particular $g_{i}(j)<g_{i}(j+1)$. At the same time, since $g_{i}(j+1) \geq \delta y_{j+1}$, the equivalence in (1) implies that $g_{i}(j) \geq g_{i}(j+1)$, and we obtain a contradiction. This proves that $M_{i}$ is a singleton.

Combining Propositions 4.1 and 4.2 leads to the following result.

Corollary 4.3 Consider some $y \in X$. For every $i \in N$, the solution $a^{i}(y)$ to the optimization problem of player $i \in N$ is unique.

Clearly, all players who are in the indifference class $\left[\ell_{i}, u_{i}\right]$ of player $i \in N$ make the same proposal. The next proposition shows that the solution to the optimization problem of a player varies continuously with $y \in X$.

Proposition 4.4 For every $i \in N$, the function $a^{i}: X \rightarrow X$ is continuous.

Proof The correspondence $\varphi: X \rightarrow X$ defined by

$$
\varphi(y)=\{x \in X \mid x \geq \delta y\}, \quad y \in X,
$$

is compact-valued and has a closed graph, so is upper hemi-continuous. To show it is lower hemi-continuous, consider $\bar{y} \in X$, a sequence $\left(y^{k}\right)_{k \in \mathbb{N}}$ in $X$ converging to $\bar{y}$, and $\bar{x} \in \varphi(\bar{y})$. We have to construct a sequence $\left(x^{k}\right)_{k \in \mathbb{N}}$ in $X$ such that $x^{k} \in \varphi\left(y^{k}\right)$ for all $k \in \mathbb{N}$, and $x^{k} \rightarrow \bar{x}$. We define

$$
x^{k}=\delta y^{k}+\alpha_{k} \max \left\{\bar{x}-\delta y^{k}, 0\right\},
$$


where

$$
\alpha_{k}=\frac{1-\delta}{\sum_{j \in N} \max \left\{\bar{x}_{j}-\delta y_{j}^{k}, 0\right\}}
$$

and $\max \left\{\bar{x}-\delta y^{k}, 0\right\}$ denotes the vector obtained by taking the component-wise maximum. The denominator in the expression for $\alpha_{k}$ is well defined since

$$
\sum_{j \in N} \max \left\{\bar{x}_{j}-\delta y_{j}^{k}, 0\right\} \geq \sum_{j \in N}\left(\bar{x}_{j}-\delta y_{j}^{k}\right)=1-\delta>0,
$$

so $\alpha_{k} \in(0,1]$.

We show next that for all $k \in \mathbb{N}$, it holds that $x^{k} \in \varphi\left(y^{k}\right)$. Clearly, it holds that $x^{k}(N)=1$ and $x^{k} \geq \delta y^{k}$.

Consider some $j, j^{\prime} \in N$ with $j \preceq j^{\prime}$.

If $\bar{x}_{j} \leq \delta y_{j}^{k}$, then $x_{j}^{k}=\delta y_{j}^{k} \leq \delta y_{j^{\prime}}^{k} \leq x_{j^{\prime}}^{k}$, so consider the case where $\bar{x}_{j}>\delta y_{j}^{k}$, so $x_{j}^{k}=\alpha_{k} \bar{x}_{j}+\left(1-\alpha_{k}\right) \delta y_{j}^{k}$.

If $\bar{x}_{j^{\prime}} \leq \delta y_{j^{\prime}}^{k}$, then

$$
x_{j^{\prime}}^{k}=\delta y_{j^{\prime}}^{k} \geq \bar{x}_{j^{\prime}} \geq \bar{x}_{j}>\alpha_{k} \bar{x}_{j}+\left(1-\alpha_{k}\right) \delta y_{j}^{k}=x_{j}^{k} .
$$

If $\bar{x}_{j^{\prime}}>\delta y_{j^{\prime}}^{k}$, then

$$
x_{j^{\prime}}^{k}=\alpha_{k} \bar{x}_{j^{\prime}}+\left(1-\alpha_{k}\right) \delta y_{j^{\prime}}^{k} \geq \alpha_{k} \bar{x}_{j}+\left(1-\alpha_{k}\right) \delta y_{j}^{k}=x_{j}^{k} .
$$

We have shown that $x^{k} \in \varphi\left(y^{k}\right)$.

Since $y^{k} \rightarrow \bar{y}$, we have that

$$
\alpha_{k}=\frac{1-\delta}{\sum_{j \in N} \max \left\{\bar{x}_{j}-\delta y_{j}^{k}, 0\right\}} \rightarrow \frac{1-\delta}{\sum_{j \in N} \max \left\{\bar{x}_{j}-\delta \bar{y}_{j}, 0\right\}}=\frac{1-\delta}{\sum_{j \in N}\left(\bar{x}_{j}-\delta \bar{y}_{j}\right)}=1
$$

so

$$
x^{k} \rightarrow \delta \bar{y}+\max \{\bar{x}-\delta \bar{y}, 0\}=\delta \bar{y}+\bar{x}-\delta \bar{y}=\bar{x} .
$$

We have shown that $\varphi$ is lower hemi-continuous.

The function $f_{i}: X \rightarrow \mathbb{R}$ defined by $f_{i}(x)=x_{i}$ is continuous. An application of the maximum theorem yields that the correspondence $\mu_{i}: X \rightarrow X$ defined by

$$
\mu_{i}(y)=\left\{\bar{x} \in \varphi(y) \mid \forall x \in \varphi(y), f_{i}(x) \leq f_{i}(\bar{x})\right\}, \quad y \in X
$$

is upper hemi-continuous. Since $\mu_{i}(y)=\left\{a^{i}(y)\right\}$ for all $y \in X$, it follows that $a^{i}$ is a continuous function. 


\section{Uniqueness of the bargaining equilibrium}

Little is known about the uniqueness of SSPE in multilateral bargaining models with unanimous agreement. It follows from the results in Kalandrakis (2006) that the most one can hope for is that the number of equilibria is odd for generic specifications of the model.

Under more special assumptions, it is possible to obtain uniqueness results, as is the case in Merlo and Wilson (1995). However, their results do not apply to our setup because the set $X$ does not lead to functions $\xi^{i}$ as required in their Assumption (A1). To see this, let $n=2$ and let the order $\preceq$ consist of the single pair $(1,2)$, so

$$
X=\left\{\left(x_{1}, x_{2}\right) \in \mathbb{R}_{+}^{2} \mid x_{1}+x_{2}=1 \text { and } x_{1} \leq x_{2}\right\} .
$$

For $i=1,2$, the function $\xi^{i}: \mathbb{R}^{2} \rightarrow \mathbb{R}$ is defined by

$$
\xi^{i}(d)=\sup \left(\left\{x_{i} \in \mathbb{R} \mid x \in X, x_{-i} \geq d_{-i}\right\} \cup\{0\}\right), \quad d \in \mathbb{R}^{2} .
$$

Thus, $\xi^{2}(d)=\min \left\{1,1-d_{1}\right\}$ if $d_{1} \leq \frac{1}{2}$, and $\xi^{2}(d)=0$ if $d_{1}>\frac{1}{2}$, violating the condition of item (b) of assumption (A1) that the function $\xi^{i}$ be continuous. Moreover, setting $x=(1 / 2,1 / 2)$ and $d=(0,0)$, we obtain a contradiction with item (cii) as $x_{1}=\xi^{1}(d)$ and $x_{2}>d_{2}$, while $x$ is an element of $X$. Neither does the set $X$ satisfy the additional Assumption (A2) required for the uniqueness result.

For the case where the set of feasible proposals is the unit interval, uniqueness results are given in Imai and Salonen (2000), Cho and Duggan (2003), Cardona and Ponsatí (2007), and Herings and Predtetchinski (2010). Imai and Salonen (2000) study the case where utility functions are either monotonically increasing or monotonically decreasing on the unit interval. Cho and Duggan (2003) prove uniqueness for quadratic utility functions and show that uniqueness does not hold for general concave utility functions. It follows from Cardona and Ponsatí (2007) that concavity together with symmetry around the peak is sufficient for uniqueness. Herings and Predtetchinski (2010) prove uniqueness for a rather general proposer selection protocol in case the utility of a player is given by the distance to some ideal point. Obviously, none of these results implies uniqueness in our setting since the multi-dimensional set $X$ cannot be generated from a 1-dimensional physical cake $C$ by utility functions satisfying the assumptions of the various uniqueness results.

In this section, we demonstrate that the bargaining equilibrium is unique by establishing that the function $f: X \rightarrow X$ defined by

$$
f(y)=\sum_{i=1}^{n} \frac{1}{n} a^{i}(y), \quad y \in X,
$$

is a contraction with contraction coefficient $\delta$, i.e., for all $y, \bar{y} \in X$ it holds that $\|f(y)-f(\bar{y})\| \leq \delta\|y-\bar{y}\|$, where $\|\cdot\|$ denotes the infinity norm. It follows directly from the definition of a bargaining equilibrium that $y \in X$ is a fixed point of $f$ if and only if $\left(a^{1}(y), \ldots, a^{n}(y)\right)$ is a bargaining equilibrium. 
Consider a player $i \in N$. We use the notation $m_{i}(y)$ for the unique threshold as defined in Proposition 4.1, so we now make the dependence on $y \in X$ explicit. For $m \in\left[u_{i}, n\right] \cap U$, we define $X_{m}^{i}=\left\{y \in X \mid m_{i}(y)=m\right\}$. The closed-form expression for $X_{m}^{i}$ is given by

$$
\begin{aligned}
& X_{m}^{i}=\left\{y \in X \mid \delta y_{m} \leq \frac{\delta y\left[\ell_{i}, m\right]+1-\delta}{m-\ell_{i}+1}<\delta y_{m+1}\right\}, \quad \text { if } m<n, \\
& X_{m}^{i}=\left\{y \in X \mid \delta y_{m} \leq \frac{\delta y\left[\ell_{i}, m\right]+1-\delta}{m-\ell_{i}+1}\right\}, \quad \text { if } m=n .
\end{aligned}
$$

On the set $X_{m}^{i}$, the function $a^{i}$ is given by

$$
a_{j}^{i}(y)= \begin{cases}\frac{\delta y\left(\left[\ell_{i}, m\right]\right)+1-\delta}{m-\ell_{i}+1}, & j \in\left[\ell_{i}, m\right], \\ \delta y_{j}, & j \in N \backslash\left[\ell_{i}, m\right] .\end{cases}
$$

We denote the closure of $X_{m}^{i}$ by $\bar{X}_{m}^{i}$. The closed-form expression for a non-empty set $\bar{X}_{m}^{i}$ is given by

$$
\begin{aligned}
& \bar{X}_{m}^{i}=\left\{y \in X \mid \delta y_{m} \leq \frac{\delta y\left[\ell_{i}, m\right]+1-\delta}{m-\ell_{i}+1} \leq \delta y_{m+1}\right\}, \quad \text { if } m<n, \\
& \bar{X}_{m}^{i}=\left\{y \in X \mid \delta y_{m} \leq \frac{\delta y\left[\ell_{i}, m\right]+1-\delta}{m-\ell_{i}+1}\right\}, \quad \text { if } m=n .
\end{aligned}
$$

Proposition 5.1 For all $i \in N$, for all $m \in\left[u_{i}, n\right] \cap U$, the function $a^{i}$ is a contraction on the set $\bar{X}_{m}^{i}$ with contraction coefficient $\delta$.

Proof We first argue $a^{i}$ to be a contraction on the set $X_{m}^{i}$ with contraction coefficient $\delta$. Take points $y$ and $\bar{y}$ in $X_{m}^{i}$. For each $j \in\left[\ell_{i}, m\right]$, we have the inequalities

$$
\begin{aligned}
\left|a_{j}^{i}(y)-a_{j}^{i}(\bar{y})\right| & =\left|\frac{\delta y\left(\left[\ell_{i}, m\right]\right)+1-\delta}{m-\ell_{i}+1}-\frac{\delta \bar{y}\left(\left[\ell_{i}, m\right]\right)+1-\delta}{m-\ell_{i}+1}\right| \\
& =\frac{\delta}{m-\ell_{i}+1}\left|y\left(\left[\ell_{i}, m\right]\right)-\bar{y}\left(\left[\ell_{i}, m\right]\right)\right| \\
& \leq \frac{\delta}{m-\ell_{i}+1} \sum_{j=\ell_{i}}^{m}\left|y_{j}-\bar{y}_{j}\right| \leq \frac{\delta}{m-\ell_{i}+1} \sum_{j=\ell_{i}}^{m}\|y-\bar{y}\|=\delta\|y-\bar{y}\| .
\end{aligned}
$$

For each $j \in N \backslash\left[\ell_{i}, m\right]$, we have

$$
\left|a_{j}^{i}(y)-a_{j}^{i}(\bar{y})\right|=\delta\left|y_{j}-\bar{y}_{j}\right| \leq \delta\|y-\bar{y}\| .
$$

We conclude that

$$
\left\|a^{i}(y)-a^{i}(\bar{y})\right\| \leq \delta\|y-\bar{y}\| .
$$


Now take points $y$ and $\bar{y}$ in $\bar{X}_{m}^{i}$. Let $\left(y^{k}\right)_{k \in \mathbb{N}}$ and $\left(\bar{y}^{k}\right)_{k \in \mathbb{N}}$ be sequences in $X_{m}^{i}$ converging to $y$ and $\bar{y}$, respectively. Then, for all $k \in \mathbb{N}$,

$$
\left\|a^{i}\left(y^{k}\right)-a^{i}\left(\bar{y}^{k}\right)\right\| \leq \delta\left\|y^{k}-\bar{y}^{k}\right\|
$$

and by taking the limit as $k \rightarrow \infty$, we have

$$
\left\|a^{i}(y)-a^{i}(\bar{y})\right\| \leq \delta\|y-\bar{y}\|,
$$

where we use the continuity of $a^{i}$ as derived in Proposition 4.4.

The next step is to extend the result of Proposition 5.1, claiming that $a^{i}$ is a contraction on each set $\bar{X}_{m}^{i}$, to a result valid for the entire domain $X$. To derive this extension, we exploit the fact that each $\bar{X}_{m}^{i}$ is a polytope.

Theorem 5.2 For all $i \in N$, the function $a^{i}$ is a contraction with contraction coefficient $\delta$.

Proof It follows from Proposition 4.2 that the collection $\left\{X_{m}^{i} \mid m \in\left[u_{i}, n\right] \cap U\right\}$ is a partition of $X$, so the collection $\left\{\bar{X}_{m}^{i} \mid m \in\left[u_{i}, n\right] \cap U\right\}$ is a cover of $X$. Since all the equalities and inequalities defining $\bar{X}_{m}^{i}$ are linear, it follows that $\bar{X}_{m}^{i}$ is a polytope.

Take points $y$ and $\bar{y}$ in $X$ and consider a straight line from $y$ to $\bar{y}$. Since all the sets $\bar{X}_{m}^{i}$ are polytopes, there exist $y^{1}, \ldots, y^{k^{\prime}}$ which are all on the line from $y$ to $\bar{y}$ and which are such that $y^{1}=y, y^{k^{\prime}}=\bar{y}$, and for all $k \in\left[1, k^{\prime}-1\right]$ there exists $m \in\left[u_{i}, n\right]$ such that both $y_{k}$ and $y_{k+1}$ belong to $\bar{X}_{m}^{i}$. We have that

$$
\left\|a^{i}(y)-a^{i}(\bar{y})\right\| \leq \sum_{k=1}^{k^{\prime}-1}\left\|a^{i}\left(y^{k}\right)-a^{i}\left(y^{k+1}\right)\right\| \leq \sum_{k=1}^{k^{\prime}-1} \delta\left\|y^{k}-y^{k+1}\right\|=\delta\|y-\bar{y}\|,
$$

where the second inequality follows from Proposition 5.1.

Theorem 5.3 The function $f$ is a contraction with contraction coefficient $\delta$.

Proof For each $y$ and $\bar{y}$, we have the following inequalities

$$
\|f(y)-f(\bar{y})\|=\left\|\sum_{i=1}^{n} \frac{1}{n}\left(a^{i}(y)-a^{i}(\bar{y})\right)\right\| \leq \sum_{i=1}^{n} \frac{1}{n}\left\|a^{i}(y)-a^{i}(\bar{y})\right\| \leq \delta\|y-\bar{y}\|,
$$

where the last inequality follows from Theorem 5.2.

The function $f$ being a contraction implies that it has a unique fixed point. Since the fixed points of $f$ are in a one-one correspondence with bargaining equilibria, we find the following corollary.

Corollary 5.4 There is a unique bargaining equilibrium. 
Table 1 The functions $a^{i}$ if $m_{1}=1$ and $m_{2}=2$

Table 2 The functions $a^{i}$ if $m_{1}=2$ and $m_{2}=2$

\begin{tabular}{ll}
\hline$a^{1}(y)$ & $a^{2}(y)$ \\
\hline $1-\delta+\delta y_{1}$ & $\delta y_{1}$ \\
$\delta y_{2}$ & $1-\delta+\delta y_{2}$ \\
\hline
\end{tabular}

\begin{tabular}{ll}
\hline$a^{1}(y)$ & $a^{2}(y)$ \\
\hline$\frac{1}{2}$ & $\delta y_{1}$ \\
$\frac{1}{2}$ & $1-\delta+\delta y_{2}$ \\
\hline
\end{tabular}

\section{Two special cases}

This section calculates the bargaining equilibrium for the cases where $n=2$ and $n=3$ and the order $\preceq$ is linear. This analysis also gives some first insights into the equilibrium choice of $m_{i}$ as well as the limit proposal when $\delta \uparrow 1$.

\subsection{Two players}

In this subsection, we assume $n=2$ and $1 \prec 2$.

We show first that there are no values of $\delta$ for which in a bargaining equilibrium it holds that $m_{1}=1$ and $m_{2}=2$.

Table 1 shows the functions $a^{i}$. At equilibrium, it holds that $a^{1}(y)+a^{2}(y)=2 y$, which can be rewritten as

$$
\left[\begin{array}{cc}
2-2 \delta & 0 \\
0 & 2-2 \delta
\end{array}\right]\left[\begin{array}{l}
y_{1} \\
y_{2}
\end{array}\right]=\left[\begin{array}{l}
1-\delta \\
1-\delta
\end{array}\right]
$$

Solving it gives $y_{1}=y_{2}=1 / 2$.

Moreover, $m_{i}$ should satisfy the inequalities presented in Proposition 4.1, so $\delta y_{1} \leq$ $a_{1}^{1}(y)<\delta y_{2}$ and $\delta y_{2} \leq a_{2}^{2}(y)$. It holds that $a_{1}^{1}(y)=1-\delta / 2>\delta / 2=\delta y_{2}$, so there is no value for $\delta$ for which $m_{1}=1$ and $m_{2}=2$.

Since a bargaining equilibrium exists by Corollary 5.4, we have for all values of $\delta$ that $m_{1}=2$ and $m_{2}=2$ in equilibrium. Table 2 depicts the corresponding functions $a^{i}$.

At equilibrium, it holds that $a^{1}(y)+a^{2}(y)=2 y$, which can be rewritten as

$$
\left[\begin{array}{cc}
2-\delta & 0 \\
0 & 2-\delta
\end{array}\right]\left[\begin{array}{l}
y_{1} \\
y_{2}
\end{array}\right]=\left[\begin{array}{c}
\frac{1}{2} \\
\frac{3}{2}-\delta
\end{array}\right] .
$$

Solving this linear system of equations gives $y_{1}=1 /(4-2 \delta)$ and $y_{2}=(3-2 \delta) /(4-$ $2 \delta$ ). It can be verified that $m_{1}$ and $m_{2}$ satisfy the inequalities of Proposition 4.1 for all values of $\delta$. Table 3 presents the bargaining equilibrium proposals and the expected division of the surplus. 
Table 3 The bargaining equilibrium proposals $p^{i}$ and the expected division of the surplus $y$

It holds that $m_{1}=m_{2}=2$

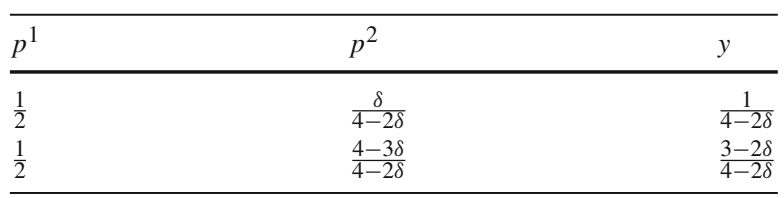

Player 2 exploits his privileged bargaining position and obtains a higher expected share in the surplus for any value of $\delta$. The difference in equilibrium payoff is highest for $\delta=0$ when equilibrium shares are given by $1 / 4$ and $3 / 4$, respectively. We are particularly interested in the limit of bargaining equilibrium proposals and the expected division of the surplus when $\delta$ tends to 1 from below. Player 1 always proposes to split the surplus equally, irrespective of the value of $\delta$. Player 2 does the same in the limit, since

$$
\lim _{\delta \uparrow 1} \frac{\delta}{4-2 \delta}=\frac{1}{2} \quad \text { and } \lim _{\delta \uparrow 1} \frac{4-3 \delta}{4-2 \delta}=\frac{1}{2} .
$$

It then follows that, in the limit, both players always receive a share equal to $1 / 2$.

\subsection{Three players}

We now analyze the case where $n=3$ and $1 \prec 2 \prec 3$.

We first compute the values of $\delta$ for which in equilibrium $m_{1}=m_{2}=m_{3}=3$. We have the following system of 12 equations and 12 unknowns.

$$
\begin{aligned}
p^{i} & =a^{i}(y), \quad i \in[1,3] \\
y & =\sum_{i \in[1,3]} \frac{1}{n} p^{i}
\end{aligned}
$$

which we can simplify as the following system in 3 equations and 3 unknowns:

$$
a^{1}(y)+a^{2}(y)+a^{3}(y)=3 y .
$$

This system can be rewritten as

$$
\left[\begin{array}{ccc}
3-2 \delta & 0 & 0 \\
0 & 3-\frac{3}{2} \delta & -\frac{1}{2} \delta \\
0 & -\frac{1}{2} \delta & 3-\frac{3}{2} \delta
\end{array}\right]\left[\begin{array}{l}
y_{1} \\
y_{2} \\
y_{3}
\end{array}\right]=\left[\begin{array}{c}
\frac{1}{3} \\
\frac{5}{6}-\frac{1}{2} \delta \\
\frac{11}{6}-\frac{3}{2} \delta
\end{array}\right],
$$

or equivalently

$$
\left[\begin{array}{ccc}
18-12 \delta & 0 & 0 \\
0 & 18-9 \delta & -3 \delta \\
0 & -3 \delta & 18-9 \delta
\end{array}\right]\left[\begin{array}{l}
y_{1} \\
y_{2} \\
y_{3}
\end{array}\right]=\left[\begin{array}{c}
2 \\
5-3 \delta \\
11-9 \delta
\end{array}\right]
$$


Table 4 The bargaining equilibrium proposals $p^{i}$ and and the expected division of the surplus $y$ if $\delta \in[0,3 / 4]$

It holds that

$m_{1}=m_{2}=m_{3}=3$

Table 5 The bargaining equilibrium proposals $p^{i}$ and the expected division of the surplus $y$ if $\delta \in(3 / 4,1)$

It holds that $m_{1}=2$ and $m_{2}=m_{3}=3$

\begin{tabular}{llll}
\hline$p^{1}$ & $p^{2}$ & $p^{3}$ & $y$ \\
\hline$\frac{1}{3}$ & $\frac{\delta}{3(3-2 \delta)}$ & $\frac{\delta}{3(3-2 \delta)}$ & $\frac{1}{3(3-2 \delta)}$ \\
$\frac{1}{3}$ & $\frac{9-7 \delta}{6(3-2 \delta)}$ & $\frac{\delta(15-11 \delta)}{6(3-\delta)(3-2 \delta)}$ & $\frac{15-11 \delta}{6(3-\delta)(3-2 \delta)}$ \\
$\frac{1}{3}$ & $\frac{9-7 \delta}{6(3-2 \delta)}$ & $\frac{25 \delta^{2}-75 \delta+54}{6(3-\delta)(3-2 \delta)}$ & $\frac{12 \delta^{2}-41 \delta+33}{6(3-\delta)(3-2 \delta)}$ \\
\hline
\end{tabular}

\begin{tabular}{llll}
\hline$p^{1}$ & $p^{2}$ & $p^{3}$ & $y$ \\
\hline$\frac{9-12 \delta+4 \delta^{2}}{18-15 \delta}$ & $\frac{3 \delta-2 \delta^{2}}{18-15 \delta}$ & $\frac{3-2 \delta^{2}}{18-15 \delta}$ & $\frac{3-2 \delta}{18-15 \delta}$ \\
$\frac{9-12 \delta+4 \delta^{2}}{18-15 \delta}$ & $\frac{9-9 \delta+\delta^{2}}{18-15 \delta}$ & $\frac{\delta}{3}$ & $\frac{1}{3}$ \\
$\frac{9 \delta-8 \delta^{2}}{18-15 \delta}$ & $\frac{9-9 \delta+\delta^{2}}{18-15 \delta}$ & $\frac{18-24 \delta+7 \delta^{2}}{18-15 \delta}$ & $\frac{9-8 \delta}{18-15 \delta}$ \\
\hline
\end{tabular}

Solving this system of equations, we obtain the unique solution given by the last column of Table 4 . To check that the proposals in Table 4 constitute an equilibrium, one has to verify the inequalities $\delta y_{3} \leq p_{3}^{1}, \delta y_{3} \leq p_{3}^{2}$, and $\delta y_{3} \leq p_{3}^{3}$. The most stringent inequality is $\delta y_{3} \leq p_{3}^{1}$. It holds if and only if $(\delta-3 / 4)(\delta-1)(\delta-2) \leq 0$, which is the case for $\delta \in[0,3 / 4]$.

We now compute the values of $\delta$ for which in equilibrium $m_{1}=2, m_{2}=3$, and $m_{3}=3$. The system of equations $6 y-2 a^{1}(y)-2 a^{2}(y)-2 a^{3}(y)=0$ can be rewritten as

$$
\left[\begin{array}{ccc}
6-5 \delta & -\delta & 0 \\
-\delta & 6-4 \delta & -\delta \\
0 & -\delta & 6-5 \delta
\end{array}\right]\left[\begin{array}{l}
y_{1} \\
y_{2} \\
y_{3}
\end{array}\right]=\left[\begin{array}{c}
1-\delta \\
2(1-\delta) \\
3(1-\delta)
\end{array}\right]
$$

Table 5 presents the solution. To check that the proposals in Table 5 constitute an equilibrium, one has to verify the inequalities $\delta y_{2} \leq p_{2}^{1}<\delta y_{3}, \delta y_{3} \leq p_{3}^{2}$, and $\delta y_{3}^{1} \leq p_{3}^{3}$. The first three inequalities are, respectively, equivalent to $(\delta-1)^{2} \geq 0$, $(\delta-1)(4 \delta-3)<0$, and $\delta-1 \leq 0$, whereas the fourth inequality is satisfied for all values of $\delta$. It follows that $\delta \in(3 / 4,1)$ gives rise to a bargaining equilibrium with $m_{1}=2, m_{2}=3$, and $m_{3}=3$.

For the case with $n=3$, the choice of $m_{i}$ depends on the value of $\delta$. For $\delta \in[0,3 / 4]$, it holds that $m_{i}=3$ for all players $i$. The equilibrium share of player 1 increases monotonically from $1 / 9$ when $\delta=0$ to $2 / 9$ when $\delta=3 / 4$. The equilibrium share of player 2 increases monotonically from $5 / 18$ when $\delta=0$ to $1 / 3$ when $\delta=3 / 4$. Player 3 can significantly exploit his more favorable bargaining position and obtains a share of $11 / 18$ when $\delta=0$ and a share of $4 / 9$ when $\delta=3 / 4$. When $\delta$ increases above $3 / 4$, the equilibrium share of player 2 stays constant at $1 / 3$, but the equilibrium share of player 1 continues to rise. In the limit when $\delta=1$, it holds that

$$
\lim _{\delta \uparrow 1}\left(\frac{9-12 \delta+4 \delta^{2}}{18-15 \delta}, \frac{9-12 \delta+4 \delta^{2}}{18-15 \delta}, \frac{9 \delta-8 \delta^{2}}{18-15 \delta}\right)=\left(\frac{1}{3}, \frac{1}{3}, \frac{1}{3}\right),
$$




$$
\begin{aligned}
& \lim _{\delta \uparrow 1}\left(\frac{3 \delta-2 \delta^{2}}{18-15 \delta}, \frac{9-9 \delta+\delta^{2}}{18-15 \delta}, \frac{9-9 \delta+\delta^{2}}{18-15 \delta}\right)=\left(\frac{1}{3}, \frac{1}{3}, \frac{1}{3}\right), \\
& \lim _{\delta \uparrow 1}\left(\frac{3-2 \delta^{2}}{18-15 \delta}, \frac{\delta}{3}, \frac{18-24 \delta+7 \delta^{2}}{18-15 \delta}\right)=\left(\frac{1}{3}, \frac{1}{3}, \frac{1}{3}\right) .
\end{aligned}
$$

As a consequence, in the limit all three players receive a share equal to $1 / 3$, irrespective of the player that proposes.

\section{Limit equilibria}

In this section, we make a first analysis of the limit of bargaining equilibria when $\delta \uparrow 1$ for an arbitrary number of players. A first result is that all players make the same proposal in the limit.

Theorem 7.1 Let $\left(\delta_{k}\right)_{k \in \mathbb{N}}$ be a sequence of continuation probabilities converging to 1 and, for $k \in \mathbb{N}$, let $p^{k}$ be the bargaining equilibrium corresponding to continuation probability $\delta_{k}$. Then for every $i, i^{\prime} \in N$ it holds that $\left\|p^{k, i}-p^{k, i^{\prime}}\right\| \rightarrow 0$.

Proof Let $y^{k}=\frac{1}{n} \sum_{j=1}^{n} p^{k, j}$ be the expected division of the surplus. Since $p^{k, j} \geq$ $\delta_{k} y^{k}$ and $\sum_{j=1}^{n} y_{j}^{k}=1$, we have that $p_{j}^{k, i} \leq \delta_{k} y_{j}^{k}+\left(1-\delta_{k}\right)$ for all $j \in N$. Therefore, it holds that $\left|p_{j}^{k, i}-p_{j}^{k, i^{\prime}}\right| \leq 1-\delta_{k}$ for every $j \in N$, and hence $\left\|p^{k, i}-p^{k, i^{\prime}}\right\| \leq 1-\delta_{k}$. By taking the limit as $k$ goes to infinity, the result follows.

We next study the limit of proposals in a bargaining equilibrium when $\delta$ converges to one from below.

Definition 7.2 The proposal $x \in X$ is a limit proposal if it is the limit of a sequence $\left(p^{k, i}\right)_{k \in \mathbb{N}}$, where $p^{k, i}$ is the proposal of some player $i \in N$ in the bargaining equilibrium corresponding to $\delta_{k}$ and $\left(\delta_{k}\right)_{k \in \mathbb{N}}$ is a sequence of continuation probabilities converging to one from below.

When studying limit proposals, the choice of the proposer $i$ is irrelevant by Theorem 7.1. Notice that although the bargaining equilibrium is unique for a given choice of $\delta_{k}$, this does not imply that the limit of such equilibria is uniquely determined.

We are interested in two questions. First, are limit proposals uniquely determined? And if so, how are they characterized? The results in Sect. 6 show that when $n=2$ or $n=3$ and the order $\preceq$ is linear, limit proposals are uniquely determined and equal to the egalitarian solution. However, demonstrating such a result for higher values of $n$ requires techniques different from brute force calculations.

The literature on multilateral bargaining with unanimous agreement has obtained a number of results on the convergence of non-cooperative equilibrium outcomes to the Nash bargaining solution. In particular, such results have been demonstrated in Hart and Mas-Colell (1996), Laruelle and Valenciano (2007), Miyakawa (2008), Kultti and Vartiainen (2010), and Britz et al. (2010) under increasingly weaker conditions. 
Definition 7.3 The Nash product is the function $\rho: \mathbb{R}_{+}^{n} \rightarrow \mathbb{R}$ defined by

$$
\rho(x)=\prod_{i \in N} x_{i}, \quad x \in \mathbb{R}_{+}^{n} .
$$

The Nash bargaining solution is the unique maximizer of the function $\rho$ on the set $X$.

Uniqueness of the Nash bargaining solution follows from convexity of the set $X$. In fact, the weaker condition of log-convexity is necessary and sufficient for uniqueness of the Nash bargaining solution, see Qin et al. (2015).

The following result establishes that the Nash bargaining solution corresponds to the egalitarian solution. The vector $e$ denotes the $n$-dimensional vector of ones.

Theorem 7.4 The Nash bargaining solution on the set $X$ is equal to $(1 / n) e$.

Proof It is easy to see that the point $(1 / n) e$ is the maximizer of the Nash product on the set $P=\left\{x \in \mathbb{R}_{+}^{n} \mid x_{1}+\cdots+x_{n}=1\right\}$. The result follows at once since all Pareto efficient points of $X$ belong to $P$.

We have found that the Nash bargaining solution coincides with the egalitarian solution for all values of $n$. Unfortunately, we cannot yet conclude that limit proposals coincide with the egalitarian solution. The reason is that all the results on convergence of multilateral bargaining with unanimous agreement to the Nash bargaining solution assume that the set of feasible utilities has a differentiable Pareto frontier, a requirement obviously not met by $X$.

Kultti and Vartiainen (2010) provide an example showing that without differentiability, the unique bargaining equilibrium may not converge to the Nash bargaining solution. Herings and Predtetchinski (2011a) show that without differentiability, the limit of bargaining equilibria may not be unique. For instance, for a set $\tilde{X}$ given by the intersection of two halfspaces,

$$
\tilde{X}=\left\{x \in \mathbb{R}^{3} \mid 2 x_{1}+x_{2}+3 x_{3} \leq 15\right\} \cap\left\{x \in \mathbb{R}^{3} \mid x_{1}+2 x_{2}+3 x_{3} \leq 15\right\},
$$

Herings and Predtetchinski (2011a) show that bargaining equilibria converge to $(3,3,2)$, whereas the Nash bargaining solution is given by $(10 / 3,10 / 3,5 / 3)$.

\section{Convergence to the Nash bargaining solution}

In this section, we will argue that irrespective of the number of players and the order $\preceq$, the limit proposal is uniquely determined and corresponds to the egalitarian solution, and therefore to the Nash bargaining solution by Theorem 7.4. The first result of this section claims that the expected equilibrium share in the surplus is strictly increasing in the order $\preceq$.

Theorem 8.1 Let $p \in X^{N}$ be a bargaining equilibrium with expected division of the surplus $y=\frac{1}{n} \sum_{j=1}^{n} p^{j}$. For $i, i^{\prime} \in N$, it holds that $i \preceq i^{\prime}$ if and only if $y_{i} \leq y_{i^{\prime}}$. 
Proof Since $y \in X$, we have that $i \sim i^{\prime}$ implies $y_{i}=y_{i^{\prime}}$.

Consider the case where $i \prec i^{\prime}$. We show that $y_{i}<y_{i^{\prime}}$. It holds that

$$
\begin{aligned}
p_{i^{\prime}}^{i^{\prime}} & =\frac{\delta}{m_{i^{\prime}}-\ell_{i^{\prime}}+1} \sum_{j=\ell_{i^{\prime}}}^{m_{i^{\prime}}} y_{j}+\frac{1-\delta}{m_{i^{\prime}}-\ell_{i^{\prime}}+1} \\
& \geq \frac{\delta}{m_{i^{\prime}}-\ell_{i^{\prime}}+1} \sum_{j=\ell_{i^{\prime}}}^{m_{i^{\prime}}} y_{i}+\frac{1-\delta}{m_{i^{\prime}}-\ell_{i^{\prime}}+1}=\delta y_{i}+\frac{1-\delta}{m_{i^{\prime}}-\ell_{i^{\prime}}+1}>\delta y_{i}=p_{i}^{i^{\prime}} .
\end{aligned}
$$

Thus, $p_{i}^{i^{\prime}}<p_{i^{\prime}}^{i^{\prime}}$ and $p_{i}^{j} \leq p_{i^{\prime}}^{j}$ for all $j \in N$ since $p^{j}$ belongs to $X$. Since $y$ is the average of the proposals $p^{1}, \ldots, p^{n}$, we find that $y_{i}<y_{i^{\prime}}$.

The next result claims that the thresholds $m_{i}$ are weakly increasing in $i$.

Theorem 8.2 Let $p \in X^{N}$ be a bargaining equilibrium with thresholds $m_{i}, i \in N$. Then it holds that $m_{1} \leq m_{2} \leq \cdots \leq m_{n}$.

Proof Suppose there is some $i \in[1, n-1]$ such that $m_{i+1}<m_{i}$, so the set $\left[\ell_{i+1}, m_{i+1}\right]$ is contained in $\left[\ell_{i}, m_{i}\right]$. We show that the vector $a^{i}(y)$ Pareto dominates the vector $a^{i+1}(y)$, which delivers the desired contradiction since the coordinates of any vector in $X$ add up to one. For every $j \in\left[\ell_{i+1}, m_{i+1}\right] \subset\left[\ell_{i}, m_{i}\right]$, we have the following chain of inequalities:

$$
a_{j}^{i+1}(y)=a_{m_{i+1}}^{i+1}(y)<\delta y_{m_{i+1}+1} \leq \delta y_{m_{i}} \leq a_{m_{i}}^{i}(y)=a_{j}^{i}(y),
$$

where $y_{m_{i+1}+1} \leq y_{m_{i}}$ follows since $m_{i+1}<m_{i}$ and the other inequality and the equations are taken from Proposition 4.1. For every $j \notin\left[\ell_{i+1}, m_{i+1}\right]$, we have

$$
a_{j}^{i+1}(y)=\delta y_{j} \leq a_{j}^{i}(y) .
$$

Thus, $a_{j}^{i+1}(y) \leq a_{j}^{i}(y)$ for every player $j$ with strict inequality whenever $j \in$ $\left[\ell_{i+1}, m_{i+1}\right]$, yielding the desired contradiction.

We show next that any player $i$ such that $i \prec n$ proposes the same share to player $i+1$ as to himself. This property can be verified to hold for $n=2$ and $n=3$ in Sect. 6.

Theorem 8.3 Let $p \in X^{N}$ be a bargaining equilibrium with thresholds $m_{i}, i \in N$. Then, for every $i \in[1, n-1]$, it holds that $m_{i}>i$.

Proof Bargaining equilibrium payoff $y$ should satisfy $y=\sum_{i \in N}(1 / n) a^{i}(y)$, with $a^{i}(y)$ given by the expression in Proposition 4.1.

Suppose $m_{i^{\prime}}=i^{\prime}$ for some $i^{\prime} \in[1, n-1]$. For $i>i^{\prime}$ it then holds that $\ell_{i}>m_{i^{\prime}}$, so

$$
\sum_{j \in\left[1, i^{\prime}\right]} a_{j}^{i}(y)=\sum_{j \in\left[1, i^{\prime}\right]} \delta y_{j} .
$$

For $i \leq i^{\prime}$ it holds that 


$$
\sum_{j \in\left[1, i^{\prime}\right]} a_{j}^{i}(y)=\sum_{j \in\left[1, i^{\prime}\right]} \delta y_{j}+(1-\delta),
$$

where we use that $m_{i^{\prime}}=i^{\prime}$ by our supposition and $m_{i} \leq m_{i^{\prime}}=i^{\prime}$ for $i \leq i^{\prime}$ by Theorem 8.2. We have that

$$
\begin{aligned}
\sum_{j \in\left[1, i^{\prime}\right]} y_{j} & =\sum_{j \in\left[1, i^{\prime}\right]} \sum_{i \in N} \frac{1}{n} a_{j}^{i}(y) \\
& =\frac{1}{n} \sum_{i \in\left[1, i^{\prime}\right]} \sum_{j \in\left[1, i^{\prime}\right]} a_{j}^{i}(y)+\frac{1}{n} \sum_{i \in\left[i^{\prime}+1, n\right]} \sum_{j \in\left[1, i^{\prime}\right]} a_{j}^{i}(y) \\
& =\frac{i^{\prime}}{n} \sum_{j \in\left[1, i^{\prime}\right]} \delta y_{j}+\frac{i^{\prime}}{n}(1-\delta)+\frac{n-i^{\prime}}{n} \sum_{j \in\left[1, i^{\prime}\right]} \delta y_{j} \\
& =\delta \sum_{j \in\left[1, i^{\prime}\right]} y_{j}+\frac{i^{\prime}}{n}(1-\delta) .
\end{aligned}
$$

By rearranging this equality, we find that $\sum_{j \in\left[1, i^{\prime}\right]} y_{j}=i^{\prime} / n$. Since $y(N)=1$, we have that the average of $y_{1}, \ldots, y_{i^{\prime}}$ is equal to the average of $y_{i^{\prime}+1}, \ldots, y_{n}$, leading to a contradiction with Theorem 8.1.

We show next that procedural fairness results in the egalitarian solution.

Theorem 8.4 The limit proposal is unique and equal to $(1 / n) e$.

Proof Consider a sequence of continuation probabilities $\left(\delta_{k}\right)_{k \in \mathbb{N}}$ converging to 1 from below. Let $p^{k}$ be the bargaining equilibrium corresponding to continuation probability $\delta_{k}$. In view of Theorem 7.1, we can assume that the sequences $\left(p^{k, i}\right)_{k \in \mathbb{N}}$ converge to the same limit $\bar{p}$ for each $i \in N$. By Theorem 8.3, it holds that for every $i \in[1, n-1]$, for every $k \in \mathbb{N}, p_{i}^{k, i}=p_{i+1}^{k, i}$, so for every $i \in[1, n-1], \bar{p}_{i}=\bar{p}_{i+1}$. We conclude that $\bar{p}_{1}=\cdots=\bar{p}_{n}$, and $\bar{p}=(1 / n) e$.

Although it has been shown in Sect. 6 that for low values of $\delta$, a player higher in the ranking $\preceq$ has a significant bargaining advantage, such an advantage disappears in the limit when $\delta$ goes to one, irrespective of the number of players and irrespective of the number and size of the indifference classes in $\preceq$.

\section{Conclusion}

In this paper, we study the effects of monotonicity constraints on bargaining outcomes. Players are ranked according to a complete order, and players higher in the ranking should not receive lower payoffs. Indifferences among players are allowed for. Monotonicity constraints result naturally in various applications in personnel economics, as well as in bankruptcy and taxation problems, and have not been taken into account in the non-cooperative bargaining literature so far. 
The crucial question is to what extent higher-ranked players can exploit their more favorable bargaining position. We prove that bargaining equilibria in the presence of monotonicity constraints are unique. Examples show that for high values of the probability of breakdown, or alternatively for low values of the discount factor, the advantage of a higher-ranked player can be substantial.

We are mainly interested in the limit of bargaining equilibria as the breakdown probability converges to zero. We are faced with the difficulty that the non-differentiabilities caused by the monotonicity constraints make previously derived limit results inapplicable and might potentially be a source of multiplicity of limit equilibria. Nevertheless, we show that in the presence of monotonicity constraints, there is a well-defined limit equilibrium which coincides with the Nash bargaining solution, similar to the differentiable case. The limit equilibrium itself corresponds to an equal division of the surplus. When players are sufficiently patient, a higher-ranked player therefore does not have a bargaining advantage.

Open Access This article is distributed under the terms of the Creative Commons Attribution 4.0 International License (http://creativecommons.org/licenses/by/4.0/), which permits unrestricted use, distribution, and reproduction in any medium, provided you give appropriate credit to the original author(s) and the source, provide a link to the Creative Commons license, and indicate if changes were made.

\section{Appendix}

Proof of Theorem 3.2 A one-shot deviation in a subgame is a single deviation by the player at the root of the subgame. It holds that $(p, A(p))$ is an SSPE if and only if there is no player having a profitable one-shot deviation. The proof of this fact is standard in the literature and is based on the optimality principle from dynamic programming.

Let $p$ be a bargaining equilibrium. We demonstrate next that no player has a profitable one-shot deviation from $(p, A(p))$. We denote the expected division of the surplus by $y=\sum_{j \in N}(1 / n) p^{j}$.

Consider a subgame starting at a history where player $i$ is the proposer, so according to $(p, A(p))$, the proposal $p^{i}$ is made by $i$ and next accepted by all players, giving rise to a share of $p_{i}^{i}$ for player $i$. Consider a one-shot deviation by player $i$ to a proposal $x \in X$. If $x$ does not belong to $\cap_{j \in N} A^{j}(p)$, it leads to an expected share $\delta y_{i}$ for player $i$. Since $\delta y_{i} \leq p_{i}^{i}$ by definition of $p^{i}$, such a deviation is not profitable. If $x$ does belong to $\cap_{j \in N} A^{j}(p)$, it holds that $x_{i} \leq p_{i}^{i}$ by definition of $p^{i}$, and again the deviation is not profitable.

Consider a subgame starting at a history where player $i$ is the responder to a proposal $x \in X$. If $x_{j} \geq \delta y_{j}$ for all $j \in N$ such that either $j=i$ or $j$ responds after player $i$, then $x$ is accepted, giving a share $x_{i}$ to player $i$. A one-shot deviation by player $i$ to rejection leads to an expected share $\delta y_{i} \leq x_{i}$ and is therefore not profitable. If $x_{i}<\delta y_{i}$ and $x_{j} \geq \delta y_{j}$ for all $j \in N$ such that $j$ responds after player $i$, then $x$ is rejected and player $i$ 's expected share is equal to $\delta y_{i}$. A one-shot deviation by player $i$ to acceptance leads to share $x_{i}<\delta y_{i}$ and is therefore not profitable. If $x_{j}<\delta y_{j}$ for some $j \in N$ such that $j$ responds after player $i$, then $x$ is rejected and player 
$i$ 's expected share is equal to $\delta y_{i}$, irrespective of player $i$ 's decision, so one-shot deviations are not profitable.

Proof of Theorem 3.3 Let $(p, A)$ be an SSPE. We denote the SSPE payoff by $y$. The proof proceeds in five steps.

(1) $\{x \in X \mid x \gg \delta y\} \subset \cap_{j \in N} A^{j}$. $^{1}$

Suppose there is $x \in X$ such that $x \gg \delta y$, but $x \notin \cap_{j \in N} A^{j}$. Let player $i$ be such that $x \notin A^{i}$ and for all players $j$ responding after player $i$ it holds that $x \in A^{j}$. Consider a subgame starting at a history where player $i$ has to respond to the proposal $x$. The expected share of player $i$ in equilibrium is equal to $\delta y_{i}$. A one-shot deviation by player $i$ to acceptance leads to a share $x_{i}>\delta y_{i}$ and is therefore profitable, a contradiction.

(2) For all $i \in N, p^{i} \in \cap_{j \in N} A^{j}$.

Suppose by way of contradiction that there is $i \in N$ such that $p^{i} \notin \cap_{j \in N} A^{j}$. Consider the subgame starting at a history where player $i$ is the proposer. Since $p^{i}$ is rejected, there is a positive probability that all players receive a share of 0 , so $\sum_{j \in N} y_{j}<1$. Moreover, since $y$ is a weighted average of vectors in $X$ and the zero vector, it holds that $y_{j} \leq y_{k}$ if $j \preceq k$. It follows that there is $x \in X$ such that $x \gg y$. The expected share of player $i$ is equal to $\delta y_{i}$ in equilibrium. Consider the one-shot deviation of player $i$ where he proposes $x$. By (1) the proposal $x$ is accepted, leading to a share $x_{i}>\delta y_{i}$ for player $i$. We have found a profitable one-shot deviation, a contradiction to $(p, A)$ being an SSPE. We have as a consequence that $y=\sum_{j \in N}(1 / n) p^{j}$.

(3) For all $i \in N, p^{i} \in \arg \max _{x \in \cap_{j \in N} A^{j}} x_{i}$.

If there would be a proposal that gives player $i$ a strictly higher share than $p^{i}$ and that is accepted by all players, player $i$ would have a profitable deviation in a subgame starting at a history where player $i$ is the proposer.

(4) $\cap_{j \in N} A^{j} \subset S=\{x \in X \mid x \geq \delta y\}$.

Suppose not. Let $x \in \cap_{j \in N} A^{j}$ and $i \in N$ be such that $x_{i}<\delta y_{i}$ and $x_{j} \geq \delta y_{j}$ for all $j \in N$ responding after $i$. Consider the subgame starting at a history where player $i$ responds to the proposal $x$. Since $x$ is accepted by $i$ and all his followers, player $i$ receives the share $x_{i}$. A one-shot deviation by player $i$ to rejection leads to an expected share $\delta y_{i}>x_{i}$, and is therefore profitable, a contradiction.

(5) For all $i \in N, p^{i} \in \arg \max _{x \in S} x_{i}$.

By (2) and (4), for all $i \in N, p^{i} \in \cap_{j \in N} A^{j} \subset S$, so $p_{i}^{i} \leq \max _{x \in S} x_{i}$. Suppose there is $i \in N$ and $\bar{x} \in S$ such that $\bar{x}_{i}>p_{i}^{i}$. The vector $\bar{y} \in \mathbb{R}^{N}$ defined by $\bar{y}_{j}=\delta y_{j}+(1-\delta) / n, j \in N$, satisfies $\bar{y} \in S$ and $\bar{y} \gg \delta y$. Let $\bar{z}$ be a strictly convex combination of $\bar{x}$ and $\bar{y}$, so $\bar{z} \in S$ and $\bar{z} \gg \delta y$. For a sufficiently small weight on $\bar{y}$ it holds that $\bar{z}_{i}>p_{i}^{i}$. By (1) it holds that $\bar{z} \in \cap_{j \in N} A^{j}$. We obtain a contradiction to $p^{i}$ being a vector in $\cap_{j \in N} A^{j}$ with maximal component $i$.

\footnotetext{
1 We use the notation $x \gg \bar{x}$ to indicate that all components of the vector $x$ strictly exceed the corresponding components of the vector $\bar{x}$, whereas $>$ is used when this property holds for at least one component.
} 


\section{References}

Aumann, R.J., Maschler, M.: Game theoretic analysis of a bankruptcy problem from the Talmud. J. Econ. Theory 36, 195-213 (1985)

Banks, J., Duggan, J.: A bargaining model of collective choice. Am. Polit. Sci. Rev. 94, 73-88 (2000)

Britz, V., Herings, P.J.J., Predtetchinski, A.: Non-cooperative support for the asymmetric nash bargaining solution. J. Econ. Theory 145, 1951-1967 (2010)

Cardona, D., Ponsatí, C.: Bargaining one-dimensional social choices. J. Econ. Theory 137, 627-651 (2007)

Cho, S.J., Duggan, J.: Uniqueness of stationary equilibria in a one-dimensional model of bargaining. J. Econ. Theory 113, 118-130 (2003)

Duggan, J.: Coalitional Bargaining Equilibria, Working, pp. 1-33 (2011)

Eraslan, H., McLennan, A.: Uniqueness of stationary equilibrium payoffs in coalitional bargaining. J. Econ. Theory 148, 2195-2222 (2013)

Hart, S., Mas-Colell, A.: Bargaining and value. Econometrica 64, 357-380 (1996)

Herings, P.J.J., Predtetchinski, A.: One-dimensional bargaining with Markov recognition probabilities. J. Econ. Theory 145, 189-215 (2010)

Herings, P.J.J., Predtetchinski, A.: On the asymptotic uniqueness of bargaining equilibria. Econ. Lett. 111, 243-246 (2011a)

Herings, P.J.J., Predtetchinski, A.: Procedurally Fair Income Taxation Schemes, METEOR Research Memorandum 11/35, Maastricht University, Maastricht, pp. 1-24 (2011b)

Imai, H., Salonen, H.: The representative nash solution for two-sided bargaining problems. Math. Social Sci. 39, 349-365 (2000)

Kalandrakis, T.: Regularity of pure strategy equilibrium points in a class of bargaining games. Econ. Theory 28, 309-329 (2006)

Kultti, K., Vartiainen, H.: Multilateral non-cooperative bargaining in a general utility space. Int. J. Game Theory 39, 677-689 (2010)

Laruelle, A., Valenciano, F.: Bargaining in committees as an extension of Nash's bargaining theory. J. Econ. Theory 132, 291-305 (2007)

Merlo, A., Wilson, C.: A stochastic model of sequential bargaining with complete information. Econometrica 63, 371-399 (1995)

Mirrlees, J.A.: An exploration in the theory of optimum income taxation. Rev. Econ. Stud. 38, 175-208 (1971)

Miyakawa, T.: Noncooperative foundation of $n$-person asymmetric Nash bargaining solution. J. Econ. Kwansei Gakuin Univ. 62, 1-18 (2008)

O’Neill, B.: A problem of rights arbitration from the Talmud. Math. Social Sci. 2, 345-371 (1982)

Qin, C.-Z., Shi, S., Tan, G.: Nash bargaining for log-convex problems. Econ. Theory 58, 413-440 (2015)

Rubinstein, A.: Perfect equilibrium in a bargaining model. Econometrica 50, 97-109 (1982)

Thomson, W.: Axiomatic and game-theoretic analysis of bankruptcy and taxation problems: a survey. Math. Social Sci. 45, 249-297 (2003) 